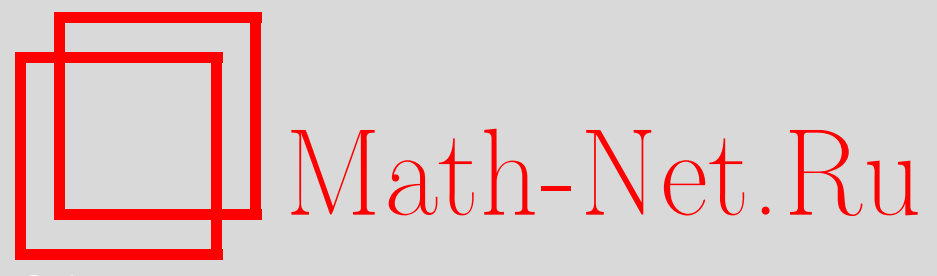

В. А. Емеличев, В. Н. Кричко, Д. П. Подкопаев, О радиусе устойчивости векторной задачи линейного булева программирования, Дискрет. матем., 2000, том 12, выпуск $2,25-30$

DOI: https://doi.org/10.4213/dm328

Использование Общероссийского математического портала Math-Net.Ru подразумевает, что вы прочитали и согласны с пользовательским соглашением http://www . mathnet.ru/rus/agreement

Параметры загрузки:

IP : 52.6 .47 .48

26 апреля 2023 г., $17: 10: 36$ 


\title{
О радиусе устойчивости векторной задачи линейного булева программирования
}

\author{
(c) 2000 г. В. А. Емеличев, В. Н. Кричко, Д. П. Подкопаев
}

Рассматривается многокритериальная задача булева программирования $\mathrm{c}$ линейными частными критериями. Найдены нижняя и верхняя достижимые оценки радиуса устойчивости множества Парето в случае, когда независимым возмущениям подвергаются одновременно и коэффициенты векторного критерия и элементы матрицы ограничений.

Работа поддержана Белорусским республиканским фондом фундаментальных исследований (проект Ф 97-266).

Под устойчивостью векторной задачи дискретной оптимизации обычно (см., например, [1-4]) понимают свойство непоявления новых паретовских оптимумов при любых изменениях параметров задачи в пределах “малой” окрестности исходных данных. Тем самым, устойчивость является дискретным аналюгом классического свойства полунепрерывности сверху в смысле Хаусдорфа точечно-множественного отображения, которое каждому набору параметров задачи ставит в соответствие множество Парето.

Хорошо известны $[1,2]$ необходимые и достаточные условия устойчивости векторной задачи целочисленного линейного программирования в случае, когда возмущению подвергаются одновременно и коэффициенты векторного критерия, и элементы матрицы ограничений. В настоящей заметке найдены количественные оценки устойчивости векторной линейной задачи булевл программирования при том же характере возмущений всех параметров задачи. Ранее в [5] была выведена формула радиуса устойчивости фиксированного паретовского оптимума векторной задачи булева программирования, а в [6] -- формула радиуса устойчивости единственного оптимума скалярной (однокритериальной) задачи булева программирования.

Будем в основном придерживаться обозначений, а по возможности и терминологии работ $[5,6]$.

Пусть $C=\left[c_{i j}\right] \in \mathbf{R}^{k \times n}, A=\left[a_{i j}\right] \in \mathbf{R}^{m \times n}, b \in \mathbf{R}^{m}, k \geqslant 1, n \geqslant 2, m \geqslant 1, \mathbf{E}^{n}-$ множество вершин единичного $n$-мерного куба $\left(\mathbf{E}^{n}=\{0,1\}^{n}\right)$.

Рассмотрим $k$-критериальную (векторную) задачу булева программирования

$$
\begin{gathered}
C x \rightarrow \max , \\
\forall i \in N_{m}\left(A_{i} x \leqslant b_{i}\right), x \in \mathbf{E}^{n},
\end{gathered}
$$

состоящую в поиске множества Парето. 
Здесь $N_{m}=\{1,2, \ldots, m\}, A_{i}=\left(a_{i 1}, a_{i 2}, \ldots, a_{i n}\right)$ и в дальнейшем нижний индекс $i$ у матрицы (вектора) будет указывать на $i$-ю строку (компоненту) соответствующей матрицы (соответствующего вектора).

Условимся для двух векторов $u, v \in \mathbf{R}^{k}$ считать

$$
\begin{aligned}
& u \leqslant v \Longleftrightarrow u_{i} \leqslant v_{i}, i \in N_{k}, \\
& u<v \Longleftrightarrow u_{i}<v_{i}, i \in N_{k} .
\end{aligned}
$$

Будем говорить, что вектор $u$ доминирует вектор $v$, если $u \geqslant v$ и $u \neq v$.

Пусть $X$ - непустое множество всех точек, удовлетворяющих системе (2), т.е. множество (допустимых) решений задачи (1)-(2). Под паретовским оптимумом задачи (1)-(2), как обычно, будем понимать элемент множества Парето

$$
P=\{x \in X: p(x)=\varnothing\},
$$

где

$$
\begin{gathered}
p(x)=\left\{x^{\prime} \in X: C\left(x-x^{\prime}\right) \leqslant \mathbf{0}, C\left(x-x^{\prime}\right) \neq \mathbf{0}\right\}, \\
\mathbf{0}=(0,0, \ldots, 0) \in \mathbf{R}^{k} .
\end{gathered}
$$

Другими словами, точка $x \in X$ является паретовским оптимумом задачи (1)-(2) тогда и только тогда, когда для всякой точки $x^{\prime} \in X$ вектор $C x^{\prime}$ не доминирует вектор $C x$.

Очевидно, что $P \neq \varnothing$ ввиду предположения $X \neq \varnothing$. В случае, когда $k=1$, множество Парето превращается в множество оптимальных решений, а наша задача - в обычную скалярную задачу булева программирования.

"Возмущенной" задачей будем называть следующую векторную задачу поиска множества Парето:

$$
\begin{gathered}
\left(C+C^{\prime}\right) x \rightarrow \max , \\
\forall i \in N_{m}\left(\left(A_{i}+A_{i}^{\prime}\right) x \leqslant b_{i}+b_{i}^{\prime}\right), x \in \mathbf{E}^{n} .
\end{gathered}
$$

Расстояние между задачами (1)-(2) и (3)-(4) определим числом

$$
\max \left\{\left\|C^{\prime}\right\|,\left\|A^{\prime}\right\|,\left\|b^{\prime}\right\|\right\},
$$

где $A^{\prime}$ - матрица со строками $A_{i}^{\prime}, i \in N_{m}, b^{\prime}=\left(b_{1}^{\prime}, b_{2}^{\prime}, \ldots, b_{m}^{\prime}\right),\|\cdot\|$ - чебышевская норма $\left(l_{\infty}\right)$ в соответствующем пространстве. Через $\|\cdot\|^{*}$ будем, как обычно, обозначать норму в сопряженном пространстве $\left(l_{1}\right)$.

По аналогии с $[5,6]$ радиусом устойчивости $k$-критериальной задачи $(1)-(2)$ назовем инфимум расстояний от этой задачи до задач вида (3)-(4), каждая из которых или не имеет решений, или имеет хотя бы один паретовский оптимум, не являющийся паретовским оптимумом задачи (1)-(2).

Радиус устойчивости задачи (1)-(2) будем обозначать через $\rho^{k}(A, b, C)$ или просто $\rho$.

В дальнейшем число $\max \left\{\left\|A^{\prime}\right\|,\left\|b^{\prime}\right\|\right\}$ будем считать расстоянием между системами (2) и (4).

Точку $x^{0} \in X$ назовем $\varepsilon$-устойчивым решением системы (2), если $x^{0}$ - решение любой системы (4), находящейся от (2) на расстоянии, не превосходящем $\varepsilon$.

ІІусть

$$
\beta(x)=\max \left\{\frac{A_{i} x-b_{i}}{\|x\|^{*}+1}: i \in N_{m}\right\} .
$$

Нам понадобятся следующие две леммы, доказанные в [6]. 
Лемма 1 ([6]). Точка $x^{0} \in X$ является $\varepsilon$-устойчивъм решением системы (2) тогда и толъко тогда, когда въполняется неравенство

$$
\varepsilon \leqslant-\beta\left(x^{0}\right)
$$

Пусть $x \in \mathbf{E}^{n} \backslash X$. Обозначим через $\beta^{\prime}(x)$ расстояние от системы (2) до ближайшей из систем (4), имеющих точку $x$ в качестве решения.

Лемма 2 ([6]). Для всякой точки $x \in \mathbf{E}^{n} \backslash X$ справедливо равенство

$$
\beta(x)=\beta^{\prime}(x) \text {. }
$$

Далее положим

$$
\begin{aligned}
\varphi^{k}(A, b, C) & =\min _{x \in \bar{P}} \max _{x^{\prime} \in \overline{\{x\}}} \min \left\{\alpha\left(x^{\prime}, x\right),-\beta\left(x^{\prime}\right)\right\}, \\
\psi^{k}(A, b, C) & =\min _{x \in \bar{P}} \max _{x^{\prime} \in \overline{\{x\}}} \max \left\{\alpha\left(x^{\prime}, x\right), \beta(x)\right\},
\end{aligned}
$$

где

$$
\begin{aligned}
\overline{\{x\}} & =\mathbf{E}^{n} \backslash\{x\}, \\
\alpha\left(x^{\prime}, x\right) & =\min \left\{\frac{C_{i}\left(x^{\prime}-x\right)}{\left\|x^{\prime}-x\right\|^{*}}: i \in N_{k}\right\} .
\end{aligned}
$$

Задачу (1)-(2) будем называть нетривиальной, если

$$
\bar{P}=\mathbf{E}^{n} \backslash P \neq \varnothing
$$

Замечание 1. Очевидно, что в случае $\bar{P}=\varnothing$ радиус устойчивости задачи (1)(2) равен инфимуму расстояний от этой задачи до задач вида (3)-(4), не имеющих решений.

Теорема 1. Для радиуса устойчивости нетривиалъной $k$-критериалъной $(k \geqslant 1)$ задачи булева программирования (1)-(2) справедливь оченки

$$
\varphi^{k}(A, b, C) \leqslant \rho^{k}(A, b, C) \leqslant \psi^{k}(A, b, C) .
$$

Доказательство. Сначала докажем правое из этих неравенств. Легко видеть, что для этого достаточно доказать справедливость неравенства

$$
\rho \leqslant \max \left\{\max _{x^{\prime} \in \overline{\{x\}}} \alpha\left(x^{\prime}, x\right), \beta(x)\right\}
$$

для любой точки $x \in \bar{P}$.

Пусть $x \in \bar{P}, \psi(x)$ - правая часть последнего неравенства. Рассмотрим два случая.

Случай 1: $x \in X$. Тогда $\beta(x) \leqslant 0$. Поэтому

$$
\psi(x)=\max \left\{\alpha\left(x^{\prime}, x\right): x^{\prime} \in \overline{\{x\}}\right\} \geqslant 0 .
$$


Рассмотрим возмущенную задачу (3)-(4), для которой $A^{\prime}=[0]_{m \times n}, b^{\prime}=$ $(0,0, \ldots, 0)^{T} \in \mathbf{R}^{m}, C^{\prime}=\left[c_{i j}^{\prime}\right]_{k \times n}-$ матрица, элементы которой для любой пары $(i, j) \in N_{k} \times N_{n}$ задаются формулой

$$
c_{i j}^{\prime}= \begin{cases}\varepsilon, & \text { если } x_{j}=1, \\ -\varepsilon, & \text { если } x_{j}=0,\end{cases}
$$

где $\varepsilon>\psi(x)$.

Тем самым, расстояние между исходной и возмущенной задачами равно $\varepsilon$. Осталось показать, что точка $x$ - паретовский оптимум нашей возмущенной задачи.

Полагая $e=(1,1, \ldots, 1)^{T} \in \mathbf{E}^{k}$, для любой точки $x^{\prime} \in \overline{\{x\}}$ легко выводим

$$
\begin{aligned}
\left(C+C^{\prime}\right)\left(x-x^{\prime}\right) & =C\left(x-x^{\prime}\right)+\varepsilon\left\|x-x^{\prime}\right\|^{*} e \\
& >C\left(x-x^{\prime}\right)+\psi(x)\left\|x-x^{\prime}\right\|^{*} e \\
& \geqslant C\left(x-x^{\prime}\right)+\alpha\left(x^{\prime}, x\right)\left\|x-x^{\prime}\right\|^{*} e \\
& =C\left(x-x^{\prime}\right)+e \min \left\{C_{i}\left(x^{\prime}-x\right): i \in N_{k}\right\} \\
& =C\left(x-x^{\prime}\right)-e \max \left\{C_{i}\left(x-x^{\prime}\right): i \in N_{k}\right\}
\end{aligned}
$$

Очевидно, что вектор, стоящий в правой части этих соотношений, имеет хотя бы одну нулевую координату. Поэтому ни при какой точке $x^{\prime} \in X$ вектор $\left(C+C^{\prime}\right) x^{\prime}$ не доминирует вектор $\left(C+C^{\prime}\right) x$. Следовательно, точка $x-$ паретовский оптимум нашей возмущенной задачи.

Случай 2: $x \in \mathbf{E}^{n} \backslash X$. Построим новую возмущенную задачу, выбрав матрицу $A^{\prime}$ и вектор $b^{\prime}$ так, чтобы точка $x$ была решением системы (4), а расстояние от этой системы до системы (2) было равно $\beta(x)$. Согласно лемме 2 это возможно. Далее матрицу $C^{\prime}$ зададим такой же, как и в случае 1 . Тем самым, расстояние между исходной и возмущенной задачами также равно числу $\varepsilon>\psi(x)$ (ввиду $\beta(x) \leqslant \psi(x)$ ).

Теперь, повторяя рассуждения предыдущего случая, вновь убеждаемся, что точка $x$ - паретовский оптимум построенной возмущенной задачи.

Далее докажем справедливость нижней оценки радиуса устойчивости

$$
\rho \geqslant \varphi^{k}(A, b, C) \text {. }
$$

Если $\varphi=\varphi^{k}(A, b, C)=0$, то неравенство (5) очевидно.

Пусть $\varphi>0$. Ясно, что для доказательства неравенства (5) в этом случае достаточно показать, что

(а) для всякого числа $\varepsilon<\varphi$ существует $\varepsilon$-устойчивое решение задачи (1)-(2);

(b) всякая точка $x \in \mathbf{E}^{n}$, не являющаяся паретовским опітимумом задачи (1)-(2), остается таковой в любой возмущенной задаче $(3)-(4)$, находящейся от задачи (1)-(2) на любом расстоянии $\varepsilon<\varphi$.

Пусть $x \in \bar{P}, 0<\varepsilon<\varphi$. Тогда существует такая точка $y \in \overline{\{x\}}$, что

$$
\varepsilon<\varphi \leqslant \max _{x^{\prime} \in\{x\}} \min \left\{\alpha\left(x^{\prime}, x\right),-\beta\left(x^{\prime}\right)\right\}=\min \{\alpha(y, x),-\beta(y)\} \leqslant-\beta(y) .
$$


Отсюда $y \in X$ и поэтому согласно лемме 1 точка $y$ является $\varepsilon$-устойчивым решением системы (2). Далее, учитывая, что $\varepsilon<\alpha(y, x)$, при $\left\|C^{\prime}\right\|=\varepsilon$ получаем

$$
\begin{aligned}
\left(C+C^{\prime}\right)(x-y) & =C(x-y)+C^{\prime}(x-y) \leqslant C(x-y)+\varepsilon\|x-y\|^{*} e \\
& <C\left(x-x^{\prime}\right)+\alpha(y, x)\|x-y\|^{*} e \\
& =C(x-y)+e \min \left\{C_{i}(y-x): i \in N_{k}\right\} \\
& =C(x-y)-e \max \left\{C_{i}(x-y): i \in N_{k}\right\} \leqslant \mathbf{0} .
\end{aligned}
$$

Поэтому вектор $\left(C+C^{\prime}\right) y$ доминирует вектор $\left(C+C^{\prime}\right) x$. Следовательно, точка $x$ не является паретовским оптимумом никакой возмущенной задачи (3)-(4), находящейся на любом расстоянии $\varepsilon<\varphi$ от исходной задачи (1)-(2).

Теорема 1 доказана.

Приведем примеры задач, демонстрирующие достижимость установленных теоремой 1 нижней и верхней оценок для радиуса устойчивости.

Пример 1. Пусть

$$
A=(1,1), \quad b=10, \quad C=\left(\begin{array}{ll}
3 & 3 \\
3 & 3
\end{array}\right) .
$$

Тогда $X=\mathbf{E}^{2}, P=\{(1,1)\}$ и согласно теореме 1

$$
\frac{8}{3} \leqslant \rho^{2}(A, b, C) \leqslant 3 .
$$

Очевидно, что радиус устойчивости нашей задачи $\rho=8 / 3$, поскольку множество Парето возмущенной задачи с параметрами

$$
A^{\prime}=\left(\frac{8}{3}+a, \frac{8}{3}+a\right), \quad b^{\prime}=-\frac{8}{3}-a, \quad C^{\prime}=\left(\begin{array}{ll}
0 & 0 \\
0 & 0
\end{array}\right)
$$

состоит из двух точек $(1,0)$ и $(0,1)$ при любом числе $a>0$.

Пример 2. Пусть

$$
A=(4,2), \quad b=3, \quad C=\left(\begin{array}{ll}
0 & 0 \\
0 & 0
\end{array}\right) .
$$

Тогда $X=P=\{(0,0),(0,1)\}, 0 \leqslant \rho^{2}(A, b, C) \leqslant 1 / 2$.

Очевидно, что ни одна из точек $(1,0)$ и $(1,1)$ не может быть решением никакой системы неравенств вида (4), находящейся на расстоянии меньшем, чем $1 / 2$, от исходной системы. Кроме того, точка $(0,0)$ является $1 / 2$-устойчивым решением неравенства $4 x_{1}+2 x_{2} \leqslant 3$. Следовательно, $\rho=1 / 2$.

Легко видеть, что утверждение теоремы 1 не имеет смысла для тривиальной задачи (1)-(2) (т.е. в случае $\bar{P}=\varnothing)$, поскольку тогда величины $\varphi^{k}(A, b, C)$ и $\psi^{k}(A, b, C)$ не определены. Этот случай рассмотрим отдельно.

Теорема 2. Пусть $\bar{P}=\varnothing$. Тогда радиус устойчивости $k$-критериалъной задачи (1)-(2) выражается формулой

$$
\rho^{k}(A, b, C)=\theta=\max \left\{-\beta(x): x \in \mathbf{E}^{n}\right\} .
$$


Доказательство. Очевидно, что $\theta \geqslant 0$. Сначала докажем неравенство $\rho \geqslant \theta$.

Если $\theta=0$, то это неравенство очевидно.

Пусть $\theta>0$. Тогда для любого числа $\varepsilon<\theta$ найдется такое решение $x^{0}$ задачи (1)-(2), что $\varepsilon<-\beta\left(x^{0}\right)$. Отсюда с учетом леммы 1 получаем, что решение $x^{0}$ является $\varepsilon$-устойчивым. Таким образом, любая задача, находящаяся от задачи (1)-(2) на расстоянии, меньшим $\theta$, имеет решение. Отсюда с учетом замечания 1 получаем неравенство $\rho \geqslant \theta$.

Теперь для доказательства теоремы осталось убедиться в справедливости неравенства $\rho \leqslant \theta$. Для этого достаточно при любом $\varepsilon>\theta$ найти систему неравенств вида (4), не имеющую решений и находящуюся от системы (2) на расстоянии $\varepsilon$.

Пусть $\varepsilon>\theta, b^{\prime}=(-\varepsilon, \ldots,-\varepsilon) \in \mathbf{R}^{m}, A^{\prime}=\left[a_{i j}^{\prime}\right]_{m \times n}-$ матрица с элементами $a_{i j}^{\prime}=\varepsilon,(i, j) \in N_{m} \times N_{n}$. Тогда для любого вектора $x \in \mathbf{E}^{n}$ с учетом неравенства $\varepsilon>-\beta(x)$ имеем

$$
\begin{aligned}
\left(A_{s}+A_{s}^{\prime}\right) x-\left(b_{s}+b_{s}^{\prime}\right) & =A_{s} x-b_{s}+\varepsilon\|x\|^{*}+\varepsilon \\
& >A_{s} x-b_{s}-\beta(x)\left(\|x\|^{*}+1\right) \\
& =A_{s} x-b_{s}-\max \left\{A_{i} x-b_{i}: i \in N_{m}\right\}=0
\end{aligned}
$$

где $s=s(x)=\operatorname{argmax}\left\{A_{i} x-b_{i}: i \in N_{m}\right\}$.

Следовательно, ни для какого вектора $x \in \mathbf{E}^{n}$ неравенство $\left(A+A^{\prime}\right) x \leqslant b+b^{\prime}$ не выполняется, т.е. система (4), находящаяся от системы (2) на расстоянии $\varepsilon>\theta$, не имеет решений. Теорема 2 доказана.

Замечание 2. Функционально-избыточными ограничениями в математическом программировании называют ограничения, добавление которых оставляет неизменным множество допустимых решений задачи. В то же время введение таких ограничений может уменьшить радиус устойчивости задачи. Действительно, очевидно, что неравенство $x_{1}+x_{2} \leqslant 2$ является функционально-избыточным ограничением в примере 1 , хотя добавление этого ограничения к прежним уменьшает радиус устойчивости с $8 / 3$ до 0.

\section{Список литературы}

1. Сергиенко И. В., Козерацкая Л. Н., Лебедева Т. Т., Исследоваяие устойчивости и параметрический анализ дискретных оптимизачионных задач. Наукова думка, Киев, 1995.

2. Козерацкая Л. Н., Лебедева Т. Т., Сергиенко И. В., Исследование устойчивости задач дискретной оптимизации. Кибериетика и системный анализ (1993) №3, 78-93.

3. Козерацкая Л. Н., Лебедева Т. Т., Сергиенко Т. И., Вопросы параметрического анализа и исследования устойчивости многокритериальных задач целочисленного линейного программирования. Кибернетика (1988) 68, №3, 41-44.

4. Емеличев В. А., Кравцов М. К., Об устойчивости в траекторных задачах векторной оптимизации. Кибернетика и системный анализ (1995) №4, 137-143.

5. Емеличев В. А., Кричко В. Н., Об устойчивости паретовского оптимума векторной задачи булева программирования. Дискретная математика (1999) 11, №4, 27-32.

6. Леонтьев В. К., Мамутов К. Х. Устойчивость решений в задачах линейного булева программирования. Журн. въч. матем. и матем. физихи (1988) 28, №10, 1475-1481.

Статья поступила 27.04.1999.

Переработанный вариант поступил 9.11.1999. 